\title{
Uma Proposta de Caracterização da Mobilidade de Usuários Sem Fio Através de Medição Real
}

\author{
Carlos A. V. Campos e Luís F. M. de Moraes
}

\begin{abstract}
Resumo-Este artigo apresenta uma nova proposta para caracterização da movimentação de usuários em ambientes sem fio, através de análise espaço-temporal microscópica da mobilidade. Essa análise tem o objetivo de investigar, quantitativa e qualitativamente, as componentes do movimento e outras medidas de interesse, as quais são utilizadas para se obter maior conhecimento do comportamento de movimentação dos usuários. Além disso, é apresentada uma metodologia para essa caracterização, com o intuito de aumentar a precisão dos resultados obtidos através do uso da proposta introduzida. Por fim, alguns resultados são apresentados com base em dados de movimentações de pedestres, capturados em cenários reais através de equipamento de GPS.
\end{abstract}

Palavras-Chave-Redes Móveis Sem Fio, Caracterização da Mobilidade, Medição.

Abstract-This paper presents a new proposal for the motion characterization of users in wireless environments, through the space-time microscopic analysis of mobility. This analysis has the objective of investigating the movement components and other measures of interest, in both a qualitative and quantitative way, which are utilized to get a better knowledge of the users motion behavior. In addition, a methodology for this characterization is presented, with the intention of increasing the accuracy of results obtained through the use of the proposal. Finally, some results based upon pedestrians motions are presented, captured in real scenarios by GPS equipment.

Keywords - Wireless Mobile Networks, Mobility, Characterization, Measurements.

\section{INTRODUÇÃO E MOTIVAÇÃO}

A representação da movimentação dos usuários é um fator importante no projeto e na avaliação de protocolos e aplicações para as redes móveis sem fio, principalmente para as redes móveis ad hoc (Mobile Ad hoc NETworks - MANETs), que são redes auto-organizáveis, ou seja, os dispositivos têm a capacidade de trocar informações entre si, em movimento, sem a necessidade de uma infra-estrutura de comunicação pré-existente. Assim, a mobilidade deve ser levada em consideração nas avaliações dos protocolos propostos para essas redes, como pode ser verificado em [1], [2], [3], [4]. Acrescenta-se ainda que uma mesma aplicação ou protocolo pode apresentar um desempenho bem diferente quando o comportamento de movimentação dos dispositivos da rede é alterado.

Os modelos de mobilidade têm sido a forma mais utilizada para representar o movimento dos dispositivos sem fio nas

Carlos A. V. Campos e Luís F. M. de Moraes, Laboratório de Redes de Alta Velocidade - RAVEL, Programa de Engenharia de Sistemas e Computação - PESC/COPPE, Universidade Federal do Rio de Janeiro UFRJ, Caixa Postal: 68.511, CEP: 21.941-972, Rio de Janeiro, RJ, Brasil, E-mails: \{beto,moraes\}@ ravel.ufrj.br. avaliações de desempenho das MANETs. Além disso, entre as técnicas de avaliação de desempenho de sistemas computacionais, a analítica e a simulação, descritas em [5], são as mais utilizadas para a avaliação dessas redes. Entretanto, vários problemas, erros, e/ou fraquezas foram encontrados nas pesquisas de avaliação das MANETs, ocasionados por inconsistências no uso dessas técnicas, como pode ser visto em [3], [6], [7], [8], [9]. Assim, avaliar o desempenho dessas redess, através da técnica de medição, é cada vez mais necessário.

Este trabalho tem o objetivo de investigar, em profundidade, o comportamento de movimentação dos dispositivos sem fio através da técnica de medição. Essa investigação será realizada por dois motivos: (i) - para se ter conhecimento do comportamento de movimentação dos dispositivos em um cenário real e; (ii) - para verificar o nível de proximidade e/ou adequação dos modelos sintéticos de mobilidade utilizados nas pesquisas, com a mobilidade dos dispositivos em cenários reais. Para isso, registros de movimentação real serão capturados e utilizados em uma investigação com o intuito de possibilitar uma caracterização da mobilidade desses dispositivos.

$\mathrm{O}$ restante do artigo está organizado da seguinte forma. $\mathrm{Na}$ seção II, são descritos os principais trabalhos relacionados com modelos de mobilidade e sobre caracterização de redes móveis. Na seção III, é apresentada um nova proposta de caracterização da movimentação de usuários que compreende uma análise das componentes dessa movimentação; e uma metodologia de coleta, filtragem e tratamento dos dados. Além disso, na seção IV, foi realizado um estudo de caso de aplicação dessa proposta e alguns resultados obtidos são descritos. Por fim, as conclusões e trabalhos futuros são apresentados na seção V.

\section{TRABALhos RELACIONADOS}

A atividade de desenvolver modelos de mobilidade está cada vez mais intensa na comunidade científica, visto que as tecnologias de redes móveis estão em constante evolução. Devido a essa evolução, existem várias propostas de classificação desses modelos e que serão descritas a seguir.

É apresentado, em [1], um mapa conceitual da representação da mobilidade usada na simulação e na análise de sistemas de comunicações sem fio. Essa representação é realizada através dos componentes: (i) - nível de aleatoriedade (determinística, híbrida ou aleatória); (ii) - nível de detalhamento (micromobilidade, macromobilidade, movimentação individual ou em grupo); (iii) - representação por simulação ou por modelagem analítica; (iv) - quantidade de dimensões da representação (1D, 2D ou 3D); e (v) - na escolha da nova direção da abordagem aleatória, pode-se utilizar diferentes regras de borda para a 
área representada. Essa representação pode ser aplicada tanto nas redes sem infra-estrutura (MANETs), quanto nas redes infra-estruturadas: redes locais sem fio e de telefonia celular.

Em [2], os modelos de mobilidade são classificados em dois tipos: mobilidade individual e mobilidade em grupo. Os modelos individuais representam o comportamento de movimentação de um dispositivo de maneira independente do restante da rede. Consiste numa modelagem mais simples e de fácil implementação, visto que a representação desses dispositivos é realizada através de eventos independentes, não tendo nenhuma correlação entre estes. Devido a tais características, esses modelos são os mais usados pelos pesquisadores para avaliação dos sistemas em redes móveis. O outro tipo de classificação é a mobilidade em grupo, onde o comportamento de movimentação dos usuários possui dependência em relação ao restante da rede. Por isso, são modelos mais complexos e de difícil implementação, e portanto, menos utilizados.

Em [3], os autores classificam a mobilidade através de três fatores principais: dependência temporal, dependência espacial e restrições geográficas. Além disso, nesse trabalho é proposto um framework para a caracterização da mobilidade baseada em métricas de mobilidade e grafos de conectividade. Uma avaliação do roteamento em MANETs, sob a influência da mobilidade, também é realizada. Apesar desse trabalho ter importantes contribuições para a comunidade científica, os resultados obtidos foram baseados somente em dados sintéticos.

Uma outra proposta, recente, de classificação é apresentada em [10], onde os modelos são classificados de maneira macroscópica e microscópica. Além disso, os autores propõe um modelo baseado em regras comportamentais de como os usuários se movimentam num cenário com obstáculos, entretanto, não foi realizada uma validação com dados reais.

$\mathrm{Na}$ literatura existem diversos modelos individuais propostos, sendo os mais utilizados: random walk e waypoint. O modelo random walk foi proposto para representar o movimento da matéria e dos seres vivos, e mais tarde, foi utilizado para a avaliação das redes de telefonia celular e MANETs, como descrito em [2]. Já o modelo waypoint é uma variação do anterior e foi usado, pela primeira vez, em [11]. Estes modelos são os mais utilizados atualmente, entretanto, nos últimos anos foram descobertas características indesejáveis, nestes modelos, evidenciando um comportamento não realístico, como mostrado em [3], [7], [12], [13].

Como exemplo de outras propostas de modelos individuais, têm-se os modelos: smooth [1], city section [2] e GIMM [4]. Recentemente, modelos mais realísticos, baseados em obstáculos [10], [14], em cenários reais [15], [16] e em registros de movimentação de usuários em redes infraestruturadas [17], [18], [19] têm sido propostos. Entretanto, esses trabalhos não têm sido realizados com o objetivo de capturar a movimentação real dos dispositivos de redes sem infra-estrutura e auto-organizáveis, para uma investigação de suas componentes e para uma validação do nível de realismo dos modelos de mobilidade. Isso é um problema em aberto e tem sido discutido pela comunidade científica, como pode ser visto no projeto de caracterização de redes sem fio, denominado CRAWDAD [20]. Sendo assim, uma nova proposta de caracterização do movimento de dispositivos em redes móveis sem fio será apresentada na próxima seção.

\section{Proposta de Caracterização da Mobilidade}

Esta proposta de caracterização é baseada em uma análise microscópica quantitativa e qualitativa das componentes do movimento dos dispositivos sem fio, bem como na relação de dependência entre essas componentes. Essa análise será aplicada em relação ao contexto do espaço físico na qual os dispositivos estão inseridos e que será denominada de análise espaço-temporal da mobilidade. Por fim, será proposta uma metodologia para o tratamento dos dados dessa caracterização.

\section{A. Análise Espaço-Temporal da Mobilidade}

Refere-se a uma investigação do comportamento espacial do movimento dos dispositivos, ou seja, está relacionada com as informações de posição do dispositivo móvel num sistema de coordenadas (métrico, latitude/longitude) em relação a um instante de tempo $\left(t_{j}\right)$. A variação desta posição relativa ao intervalo de tempo $\left\{t_{1}, t_{T}\right\}$, permitirá a observação do valor das seguintes componentes do movimento: velocidade, aceleração e ângulo de mudança de direção; e ainda de algumas medidas de interesse com base nessas componentes.

A partir do valor dessas componentes e medidas, a análise espaço-temporal terá como objetivo identificar as características do movimento de dispositivos em cenários específicos. Além disso, essas informações poderão possibilitar o desenvolvimento de modelos sintéticos de mobilidade mais realistas. Assim, as componentes e as medidas do movimento utilizadas nessa análise serão definidas a seguir.

Seja $\vec{P}_{i}\left(t_{j}\right)$ o vetor que representa a posição do $i$-ésimo dispositivo, no instante $t_{j}(j=1,2, \ldots, T-1, T)$. Definindo por $x_{i}\left(t_{j}\right)$ e $y_{i}\left(t_{j}\right)$ as componentes de $\vec{P}_{i}\left(t_{j}\right)$ nas direções $\vec{x}$ e $\vec{y}$, respectivamente, no instante $t_{j}$, pode-se escrever:

$$
\vec{P}_{i}\left(t_{j}\right)=\left(x_{i}\left(t_{j}\right), y_{i}\left(t_{j}\right)\right)
$$

onde $x_{i}\left(t_{j}\right)$ e $y_{i}\left(t_{j}\right)$ são números reais e a unidade de medida de $\vec{P}_{i}\left(t_{j}\right)$ e de suas componentes é dada em metros $(m)$ quando o movimento for de pedestres ou $(\mathrm{km})$ para veículos.

Ângulo de mudança de direção - $\alpha_{i}\left(t_{j}\right)$ : é definido como sendo o ângulo de mudança de direção da movimentação do $i$-ésimo dispositivo no instante de tempo $\left(t_{j}\right)$, dado que no instante $\left(t_{j-1}\right)$ o dispositivo estava na posição $\vec{P}_{i}\left(t_{j-1}\right)$. Esse ângulo é calculado pela aplicação da Lei dos Cossenos, e essa aplicação pode ser verificada através da relação entre as componentes geométricas, ilustradas na figura 1 .

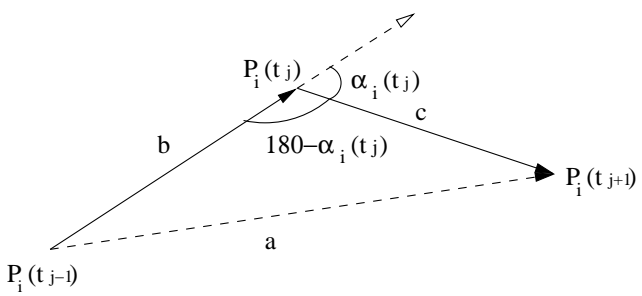

Fig. 1. Componentes geométricas de um movimento qualquer

Nessa figura, as variáveis $a, b$ e $c$ assumem o valor do módulo do vetor diferença das posições do $i$-ésimo dispositivo no tempo. Assim, $a=\left|\vec{P}_{i}\left(t_{j+1}\right)-\vec{P}_{i}\left(t_{j-1}\right)\right|, b=\mid \vec{P}_{i}\left(t_{j}\right)-$ 
$\vec{P}_{i}\left(t_{j-1}\right) \mid$ e $c=\left|\vec{P}_{i}\left(t_{j+1}\right)-\vec{P}_{i}\left(t_{j}\right)\right|$, respectivamente. Assim, $\alpha_{i}\left(t_{j}\right)$ é descrito da seguinte maneira:

$$
\alpha_{i}\left(t_{j}\right)=\arccos \left(\frac{a^{2}-b^{2}-c^{2}}{2 b c}\right)
$$

onde a unidade de medida de $\alpha_{i}\left(t_{j}\right)$ é dada em graus $\left(^{\circ}\right)$.

Velocidade do dispositivo - $V_{i}\left(t_{j}\right)$ : é a velocidade escalar do $i$-ésimo dispositivo no instante de tempo $\left(t_{j}\right)$ e é calculada por:

$$
V_{i}\left(t_{j}\right)=\frac{\sqrt{\left(x_{i}\left(t_{j}\right)-x_{i}\left(t_{j-1}\right)\right)^{2}+\left(y_{i}\left(t_{j}\right)-y_{i}\left(t_{j-1}\right)\right)^{2}}}{t_{j}-t_{j-1}}
$$

onde a unidade de medida de $V_{i}\left(t_{j}\right)$ é dada em metros por segundo $(\mathrm{m} / \mathrm{s})$ quando o movimento for de pedestres ou $(\mathrm{km} / \mathrm{h})$ para veículos.

Além da observação dos valores de cada componente do movimento, é possível verificar o comportamento da variação desse valores em relação ao tempo, ou seja, a variação da velocidade, que é a própria aceleração do movimento, e a variação do ângulo de mudança de direção, em relação ao instante de tempo $\left(t_{j}\right)$. Sendo assim, essas medidas serão definidas a seguir.

Variação da velocidade - $\Delta V_{i}\left(t_{j}\right)$ : é a aceleração do movimento e pode ser definida como sendo a variação no valor da velocidade escalar do $i$-ésimo dispositivo no instante $t_{j}$ em relação ao instante anterior $t_{j-1}$. Assim, essa aceleração é dada por:

$$
\Delta V_{i}\left(t_{j}\right)=V_{i}\left(t_{j}\right)-V_{i}\left(t_{j-1}\right)
$$

Variação do ângulo de mudança de direção - $\Delta \alpha_{i}\left(t_{j}\right)$ : é a mudança no valor do ângulo da direção do $i$-ésimo dispositivo no instante $t_{j}$ em relação ao instante anterior $t_{j-1}$. De acordo com a definição, $\Delta \alpha_{i}\left(t_{j}\right)$ é descrita da seguinte maneira:

$$
\Delta \alpha_{i}\left(t_{j}\right)=\alpha_{i}\left(t_{j}\right)-\alpha_{i}\left(t_{j-1}\right)
$$

Nessa caracterização, os valores positivos e negativos assumidos por $\Delta \alpha_{i}\left(t_{j}\right)$ serão analisados conjuntamente. Com isso, será usado somente o módulo de $\Delta \alpha_{i}\left(t_{j}\right)$ na análise.

Caso o valor do módulo das variações na velocidade e no ângulo de mudança de direção seja muito grande, isso poderá provocar modificações bruscas no movimento dos dispositivos sem fio e, portanto, essas variações também serão alvo de investigação na análise espaço-temporal. Sendo assim, serão definidas as medidas de interesse: acelerações e desacelerações bruscas, tempo de pausa no movimento e variações abruptas na mudança de direção da seguinte maneira.

Aceleração brusca - $A b_{i}\left(t_{j}\right)$ : é definida como sendo um aumento acentuado no valor da velocidade escalar do $i$-ésimo dispositivo entre dois instantes de tempo consecutivos, ou seja, é quando a variação da velocidade $\Delta V_{i}\left(t_{j}\right)$ é positiva e maior que um limiar. Assim, será utilizado $\gamma$ para representar esse limiar, isto é, o valor que limita superiormente o conjunto de valores da variação da velocidade de um dispositivo qualquer. Esse limiar é parametrizável de acordo com o tipo de usuário observado. Dessa maneira, caso o valor de $\Delta V_{i}\left(t_{j}\right)$ seja positivo e maior que $\gamma$, então $\Delta V_{i}\left(t_{j}\right)$ é considerada uma aceleração brusca e, portanto, $A b_{i}\left(t_{j}\right)=1$, senão $A b_{i}\left(t_{j}\right)=0$. Deste modo, $A b_{i}\left(t_{j}\right)$ é dada por:

$$
A b_{i}\left(t_{j}\right)=\left\{\begin{array}{lll}
1, & \text { se } & \Delta V_{i}\left(t_{j}\right)>\gamma \\
0, & \text { se } & \Delta V_{i}\left(t_{j}\right) \leq \gamma
\end{array}\right.
$$

onde, $\Delta V_{i}\left(t_{j}\right)>0$.

Número de acelerações bruscas - $N A b_{i}$ : é a quantidade de vezes em que a variação positiva da velocidade do $i$-ésimo dispositivo, no intervalo de tempo $\left\{t_{0}, T\right\}$ foi maior que o limiar $\gamma$, assim tem-se:

$$
N A b_{i}=\sum_{j=1}^{T} A b_{i}\left(t_{j}\right)
$$

Número total de acelerações bruscas - $N A b$ : é o número total de mudanças bruscas observadas no movimento de todos os dispositivos analisados num determinado cenário. Essa medida é dada a seguir:

$$
N A b=\sum_{i=1}^{n} N A b_{i}
$$

Desaceleração brusca - $D b_{i}\left(t_{j}\right)$ : é um decremento acentuado no valor da velocidade escalar do $i$-ésimo dispositivo entre dois instantes de tempo consecutivos $\left(t_{j-1}\right.$ e $\left.t_{j}\right)$. Assim, como na descrição de $A b_{i}\left(t_{j}\right)$, esse decremento é identificado quando a variação da velocidade $\Delta V_{i}\left(t_{j}\right)$ é negativa e menor que $-\gamma$. Deste modo, $D b_{i}\left(t_{j}\right)$ é dada por:

$$
D b_{i}\left(t_{j}\right)= \begin{cases}1, & \text { se } \quad \Delta V_{i}\left(t_{j}\right)<-\gamma \\ 0, & \text { se } \Delta V_{i}\left(t_{j}\right) \geq-\gamma\end{cases}
$$

onde, $\Delta V_{i}\left(t_{j}\right)<0$.

Número de desacelerações bruscas - $N D b_{i}$ : é o número de vezes em que um decremento acentuado na velocidade do $i$-ésimo dispositivo ocorreu durante o intervalo de tempo analisado $\left\{t_{0}, T\right\}$. Essa medida é dada na equação abaixo:

$$
N D b_{i}=\sum_{j=1}^{T} D b_{i}
$$

Número total de desacelerações bruscas - $N D b$ : representa a quantidade total de ocorrências de desacelerações bruscas de todos os dispositivos analisados e é dada pela seguinte equação:

$$
N D b=\sum_{i=1}^{n} N D b_{i}
$$

Tempo de pausa $-T p_{i}\left(t_{j}\right)$ : é dado pelo intervalo de instantes consecutivos de tempo em que o $i$-ésimo dispositivo permanece parado a partir do instante $t_{j}$ até o instante $t_{k}$, ou seja, a posição $P_{i}\left(t_{j}\right)$ é igual a $P_{i}\left(t_{k}\right)$, onde $j<k \leq T$. Logo, $T p_{i}\left(t_{j}\right)$ é dado por:

$T p_{i}\left(t_{j}\right)=t_{k}-t_{j}, \quad$ se $\quad P_{i}\left(t_{j}\right)=P_{i}\left(t_{k}\right) \& P_{i}\left(t_{k}\right) \neq P_{i}\left(t_{k+1}\right)$

então, a variável $p_{i}\left(t_{j}\right)$ que representa a ocorrência de pausas no movimento do $i$-ésimo dispositivo no instante $t_{j}$, assumirá o valor (um) se $T p_{i}\left(t_{j}\right)$ for diferente de zero ou assumirá o valor (zero) para o caso contrário. 
Número de pausas no movimento - $N p_{i}$ : é a quantidade de ocorrências de pausas no movimento do $i$-ésimo dispositivo durante o intervalo de tempo $\left\{t_{0}, T\right\}$. Uma pausa ocorre quando o valor da velocidade do dispositivo é igual a zero. A seguir, essa medida é definida:

$$
N p_{i}=\sum_{j=1}^{T} p_{i}\left(t_{j}\right)
$$

Número total de pausas no movimento - $N P$ : é o número de todas as pausas no movimento de todos os dispositivos observados num cenário especifico. $N P$ é calculado da seguinte maneira:

$$
N P=\sum_{i=1}^{n} N p_{i}
$$

Mudança brusca de direção - $M b \alpha_{i}\left(t_{j}\right)$ : é quando o módulo da variação no ângulo de mudança de direção do $i$-ésimo dispositivo, no tempo $t_{j}$, for maior que o limiar $\lambda$, que também é parametrizável de acordo com o tipo de usuário observado. Dessa forma, $M b \alpha_{i}\left(t_{j}\right)$ recebe o valor (um), se $\left|\Delta \alpha_{i}\left(t_{j}\right)\right|>\lambda$ ou $M b \alpha_{i}\left(t_{j}\right)$ recebe o valor (zero), se $\left|\Delta \alpha_{i}\left(t_{j}\right)\right| \leq \lambda$. Assim, $M b \alpha_{i}\left(t_{j}\right)$ é descrita da seguinte maneira:

$$
M b \alpha_{i}\left(t_{j}\right)=\left\{\begin{array}{lll}
1, & \text { se } & \left|\Delta \alpha_{i}\left(t_{j}\right)\right|>\lambda \\
0, & \text { se } & \left|\Delta \alpha_{i}\left(t_{j}\right)\right| \leq \lambda
\end{array}\right.
$$

Número de mudanças bruscas de direção - $N M b \alpha_{i}$ : é definido pela quantidade de ocorrências de $M b \alpha_{i}\left(t_{j}\right)$, na qual, $\left|\Delta \alpha_{i}\left(t_{j}\right)\right|>\lambda$ e é dado pela equação:

$$
N M b \alpha_{i}=\sum_{j=1}^{T} M b \alpha_{i}\left(t_{j}\right)
$$

Número total de mudanças bruscas de direção - $N M b \alpha$ : é a quantidade total de mudanças bruscas de direção de todos os dispositivos analisados num cenário específico. Essa medida é dada na equação abaixo:

$$
N M b \alpha=\sum_{i=1}^{n} N M b \alpha_{i}
$$

Após a definição de todas essas variáveis que servirão para quantificar o valor das componentes do movimento de um ou vários dispositivos em um intervalo de tempo, será definido agora, com base nas equações: (2), (3), (4) e (12); a média do ângulo de mudança de direção, da velocidade, da aceleração e do tempo de pausa, respectivamente.

Média do Ângulo de Mudança de Direção - $E\left(\alpha_{i}\right)$ : é definida pela soma dos ângulos de mudança de direção do $i$ ésimo dispositivo durante o intervalo de tempo $\left\{t_{1}, t_{T}\right\}$, dividido pela quantidade de amostras desses ângulos computadas. Sendo assim, essa medida é descrita abaixo:

$$
E\left(\alpha_{i}\right)=\frac{1}{T} \sum_{j=1}^{T} \alpha_{i}\left(t_{j}\right)
$$

Velocidade Média - $E\left(V_{i}\right)$ : é dada pela soma das velocidades do $i$-ésimo dispositivo durante o intervalo de tempo $\left\{t_{1}, t_{T}\right\}$, dividido pela quantidade de amostras da velocidade. Como cada amostra é obtida no próximo instante de tempo $\left(t_{j+1}\right)$, então a cardinalidade do conjunto das amostras é a própria cardinalidade do intervalo de tempo $\left\{t_{1}, t_{T}\right\}$. Assim, essa medida é descrita na equação a seguir.

$$
E\left(V_{i}\right)=\frac{1}{T} \sum_{j=1}^{T} V_{i}\left(t_{j}\right)
$$

Aceleração Média - $E\left(\Delta V_{i}\right)$ : é definida pela soma de todas as variações positivas da velocidade do $i$-ésimo dispositivo durante o intervalo de tempo $\left\{t_{1}, t_{T}\right\}$, dividido pela quantidade de amostras computadas. Essa medida é dada por.

$$
E\left(\Delta V_{i}\right)=\frac{1}{T} \sum_{j=1}^{T} \Delta V_{i}\left(t_{j}\right)
$$

Tempo Médio de Pausa - $E\left(T p_{i}\right)$ : é a soma de todos os tempos de pausa ocorridos no movimento do $i$-ésimo dispositivo durante o intervalo $\left\{t_{1}, t_{T}\right\}$, dividido pela quantidade de amostras desses tempos de pausa. Sendo assim, tem-se:

$$
E\left(T p_{i}\right)=\frac{1}{T} \sum_{j=1}^{T} T p_{i}\left(t_{j}\right)
$$

\section{B. Metodologia de Caracterização da Mobilidade}

Para que a proposta de análise da mobilidade, apresentada anteriormente, seja aplicada em registros de movimentação de maneira precisa, é necessário o uso de uma metodologia de manipulação dos dados a serem analisados.

Esta metodologia será composta por etapas que possuem uma alta dependência em relação as anteriores. Com isso, a execução cronológica dessas etapas será primordial para a eficácia da caracterização. Desta forma, estas etapas que serão descritas detalhadamente a seguir, são: definição dos cenários de observação, coleta e tratamento dos dados, e a análise estatística dos dados.

1) Definição dos cenários de observação: A metodologia proposta neste trabalho para caracterização da mobilidade é de uso geral e de grande abrangência, entretanto, é importante especificar o cenário na qual a movimentação será investigada.

Sendo assim, deve ser levado em consideração os seguintes parâmetros para cada cenário: tamanho da área em que se encontra o cenário, quantidade de dispositivos e a densidade da rede, que é dada pela razão entre a quantidade de dispositivos e o tamanho da área onde se encontra a rede sem fio.

Como exemplos de cenários ou situações de uso de redes móveis sem fio têm-se: pedestres e veículos se movimentando num centro urbano, tráfego de veículos numa rodovia, operações de busca e salvamento, o uso em áreas rurais, aplicações militares, pessoas dentro de prédios, entre outros. Todos esses cenários podem ser considerados, contudo, restrições quanto ao uso destes podem ser impostas. A seguir, a etapa de coleta e tratamento dos dados será descrita.

2) Coleta e tratamento dos dados: Após a definição do cenário é importante definir os procedimentos de captura e tratamento dos dados. Para isso, devem ser escolhidas amostras independentes, ou seja, cada amostra deve representar o movimento de usuários distintos. 
Para obter dados para essa análise é necessário a utilização de um sistema de localização. Um dos sistemas mais conhecidos e utilizados para isso é o GPS (Global Positioning System), que permite a captura da posição real do dispositivo. Dentro do contexto desse trabalho, é necessário comentar algumas características desejáveis que os GPS devem possuir para que a análise espaço-temporal seja realizada corretamente, pois o procedimento de cálculo das coordenadas de localização do dispositivo podem conter erros gerados pela variação do nível de precisão do GPS utilizado. O intervalo desses erros pode variar de centímetros até dezenas de metros, assim, quanto menor for o erro, mais precisas serão as coordenadas da localização do dispositivo.

Uma vez capturados os dados, será necessário a filtragem das amostras, que é a escolha dos dados efetivamente utilizados na caracterização. Com isso deve ser considerado o intervalo de tempo das amostras e de suas componentes. Também devem ser retirados dados inconsistentes das amostras, como, erros de gravação nos arquivos e valores discrepantes causados pelo erro no cálculo da posição. Esse erro pode ser causado pelo problema de múltiplos caminhos do sinal recebido pelo receptor GPS quando próximo de prédios e construções, e pela interferência da copa das árvores e de objetos localizados na área de visada à constelação de satélites. Após a captura e filtragem, tem-se a fase da análise estatística. Essa fase será descrita em detalhes a seguir.

3) Análise estatística dos dados: Com o objetivo de observar e ter mais conhecimento, quantitativo e qualitativo dos dados, deve ser aplicado um tratamento estatístico nas componentes do movimento e nas medidas de interesse da análise espaço-temporal introduzida na subseção anterior.

Esse tratamento consiste em calcular várias medidas estatísticas, como, desvio-padrão, variância, auto-correlação das componentes, correlação entre as componentes, frequência relativa e função de distribuição cumulativa empírica (empirical cumulative distribution function - ecdf) dessas medidas e componentes da mobilidade. Além disso, os resultados de todas essas medidads deverão ser obtidos com base em um determinado intervalo e um nível de confiança, de modo que esses resultados possam ser interpretados de maneira correta.

A partir da descrição e especificação destas etapas, acreditase que esta metodologia possibilitará a execução das tarefas de quantificação e qualificação das componentes e das medidas de interesse da mobilidade de maneira mais precisa e correta, como é recomendado em [6], permitindo assim, uma análise mais confiável da mobilidade de dispositivos sem fio.

Como grandes resultados buscados com a aplicação dessa proposta de caracterização, têm-se:

- A possibilidade de obtenção de informações mais profundas e detalhadas das componentes da mobilidade, capturadas de cenários reais ou de modelos de mobilidade;

- O fornecimento de dados importantes para o desenvolvimento de novos modelos sintéticos de mobilidade contendo características mais realistas.

\section{Aplicação da Proposta de Caracterização}

Como estudo de caso para ilustrar uma possível aplicação de uso da metodologia proposta neste trabalho, esta seção apresenta um conjunto de experimentos onde foram coletados registros de movimentação de pedestres num cenário real, bem como, a avaliação dos dados coletados. Assim, nas seções seguintes, serão descritos os detalhes destes experimentos e alguns resultados preliminares obtidos serão apresentados.

\section{A. Descrição dos Experimentos}

Os experimentos foram realizados no estacionamento do Centro de Tecnologia da UFRJ. Para a coleta do movimento foi usado um GPS de alta precisão da marca Trimble, modelo GEO XM, cujo erro de localização é de aproximadamente 0,4 $m$. O tempo de duração de cada experimento foi de $700 s$ e o intervalo entre coletas da posição do dispositivo foi de $1 \mathrm{~s}$.

Após coletados os dados e tratados conforme as etapas 1 e 2 da metodologia proposta, as seguintes medidas foram obtidas: velocidade média, aceleração média, média do ângulo de mudança de direção, tempo médio de pausa, freqüência relativa e a função de distribuição cumulativa empírica (ecdf) da velocidade instantânea e do ângulo de mudança de direção.

\section{B. Resultados Preliminares Obtidos}

Na Tabela I, é mostrada a média das componentes: velocidade, aceleração, ângulo de mud. de direção e tempo de pausa. TABELA I

COMPONENTES E MEDIDAS DE INTERESSE DA MOBILIDADE

\begin{tabular}{|c|c|}
\hline Medidas de Interesse & Dados Obtidos \\
\hline Velocidade Média & $1,0374 \mathrm{~m} / \mathrm{s}$ \\
\hline Aceleração Média & $0,0015 \mathrm{~m} / \mathrm{s}^{2}$ \\
\hline Média do Ângulo de Mud. de Dir. & $20,2781^{\circ}$ \\
\hline Tempo Médio de Pausa & $0,0058 \mathrm{~s}$ \\
\hline
\end{tabular}

Como mencionado em [7], [8], [13], [16] é importante investigar as características das distribuições das componentes da mobilidade. Deste modo, são mostrados nas figuras 2 e 3, o histograma da frequiência relativa e a distribuição cumulativa empírica (ecdf) da velocidade instantânea, respectivamente.

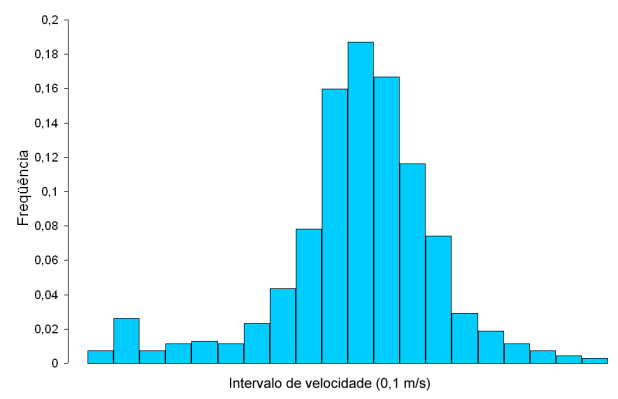

Fig. 2. Histograma da velocidade instantânea

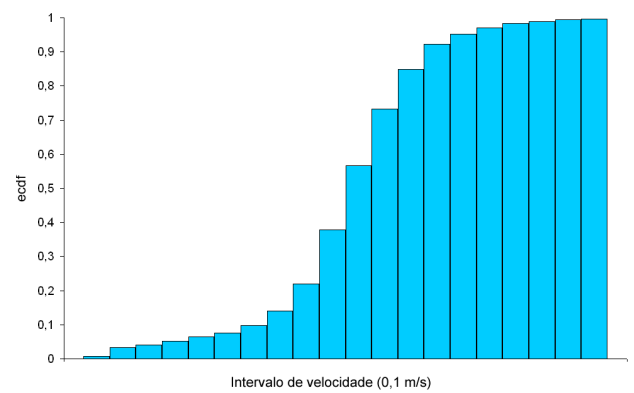

Fig. 3. Distribuição cumulativa empírica da velocidade instantânea 
Através destas figuras, pode-se perceber uma semelhança destas curvas com o comportamento da distribuição Normal, entretanto, uma investigação mais criteriosa deve ser realizada para que isso possa ser concluído.

Nas figuras 4 e 5, são ilustrados o histograma da freqüência relativa e a distribuição cumulativa empírica (ecdf) do ângulo de mudança de direção, respectivamente, computadas.

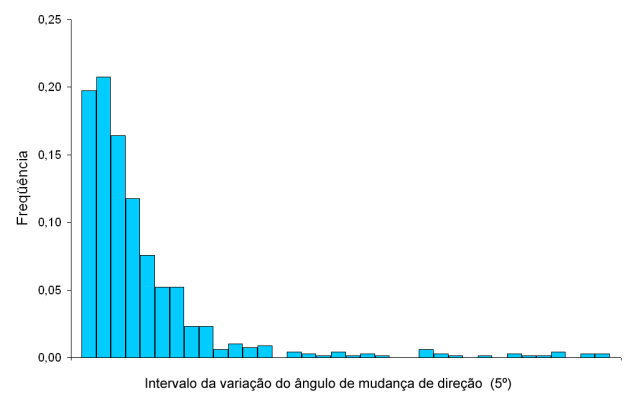

Fig. 4. Histograma do ângulo de mudança de direção

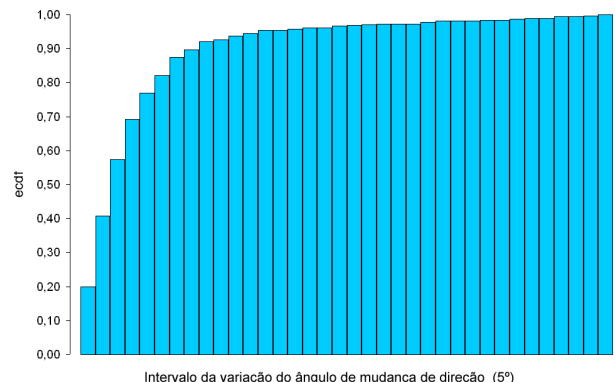

Fig. 5. Distribuição cumulativa empírica do ângulo de mudança de direção

Pode-se perceber, através destas figuras, uma semelhança desta curvas com o comportamento da distribuição Gamma, contudo, isto só pode ser concluído após a aplicação de métodos numéricos, descritos em [5], nesses dados.

Os resultados apresentados nesta seção, onde foi investigado o movimento de pedestres em um campus universitário, são preliminares e apenas motivam o uso da análise e da metodologia proposta. Para se obter mais informações sobre comportamento da mobilidade nesse cenário, é necessário a completa aplicação das medidas estatísticas da etapa 3, nos dados coletados.

\section{Conclusões e Trabalhos Futuros}

Neste artigo, é apresentada uma nova proposta de caracterização da mobilidade de dispositivos, baseada numa análise criteriosa das componentes do movimento de usuários em um ambiente sem fio. Para isso, foram definidas e utilizadas várias medidas de interesse, com o objetivo de se obter informações mais detalhadas sobre a mobilidade. Além disso, foi realizado um estudo de caso onde dados capturados do movimento de pedestres, num campus universitário, foram analisados e informações desses dados foram obtidas.

Dessa maneira, acredita-se que esta proposta seja útil para a obtenção de informações sobre o comportamento de movimentação dos usuários sem fio, para a avaliação e comparação dos modelos de mobilidade com dados reais. Além disso, esta caracterização pode fornecer dados importantes para o desenvolvimento de novos modelos de mobilidade específicos e próximos de uma realidade a ser representada por simulações em redes móveis.
Como trabalhos futuros, pretende-se: (i) - obter as medidas estatísticas, mencionadas na seção III-B.3, para conjuntos de dados maiores e mais representativos; (ii) - identificar a proximidade de alguma função de distribuição de probabilidade com o comportamento das componentes dos dados coletados, através do uso dos métodos numéricos: Maximum Likelihood Estimation (MLE) e Kolmogorov-Smirnov [5]; e (iii) - comparar os dados coletados de cenários reais com os seguintes modelos de mobilidade: waypoint, smooth, GIMM e o proposto em [10].

\section{REFERÊNCIAS}

[1] C. Bettstetter, "Mobility Modeling in Wireless Networks: Categorization, Smooth Movement, and Border Effects," ACM Mobile Computing and Communications Review, vol. 5, no. 3, 2001.

[2] T. Camp, J. Boleng, and V. Davies, "A Survey of Mobility Models for Ad Hoc Network Research," Wireless Communications and Mobile Computing (WCMC), vol. 2, no. 5, pp. 483-502, 2002.

[3] F. Bai, N. Sadagopan, and A. Helmy, "The IMPORTANT framework for analyzing the Impact of Mobility on Performance Of RouTing protocols for Ad hoc NeTworks," Elsevier Ad Hoc Net., vol. 1, pp. 383-403, 2003.

[4] C. A. V. Campos and L. F. M. de Moraes, "A Markovian Model Representation of Individual Mobility Scenarios in Ad Hoc Networks and Its Evaluation," EURASIP Journal on Wireless Communications and Networking, vol. 2007, 2007, 14 pages.

[5] R. Jain, The Art of Computer Systems Performance Analysis. New York, NY, USA: John Wiley and Sons, 1991.

[6] S. Kurkowski, T. Camp, and M. Colagrosso, "MANET Simulation Studies: the Incredibles," SIGMOBILE Mobile Computing and Communications Review, vol. 9, no. 4, pp. 50-61, 2005.

[7] J.-Y. Le Boudec and M. Vojnovic, "Perfect Simulation and Stationarity of a Class of Mobility Models," in Proc. of the INFOCOM'05, Miami, USA, 2005, pp. 72-79.

[8] J. Yoon, M. Liu, and B. Noble, "A General Framework to Construct Stationary Mobility Models for the Simulation of Mobile Netwoks," IEEE Trans. Mob. Comput., vol. 5, no. 7, pp. 860-871, jul 2006.

[9] S. Kurkowski, T. Camp, and W. Navidi, "Two Standards for Rigorous MANET Routing Protocol Evaluation," in Proc. of the IEEE MASS'06, Vancouver, Canada, oct 2006, pp. 256-266.

[10] F. Legendre, V. Borrel, M. Dias De Amorim, and S. Fdida, "Reconsidering Microscopic Mobility Modeling for Self-organizing Networks," IEEE Network Magazine, vol. 20, no. 6, pp. 4-12, 2006.

[11] D. B. Johnson and D. A. Maltz, "Dynamic Source Routing in Ad hoc Wireless Networks," Mobile Computing, 1996.

[12] M. L. J. Yoon and B. Noble, "Random Waypoint Considered Harmful," in Proc. of the IEEE INFOCOM'03, San Francisco, USA, apr 2003, pp. 1312-1321.

[13] W. Navidi and T. Camp, "Stationary Distributions for the Random Waypoint Mobility Model," IEEE Transactions on Mobile Computing, vol. 3, no. 1, pp. 99-108, 2004.

[14] A. Jardosh, E. M. Belding-Royer, K. C. Almeroth, and S. Suri, "Towards Realistic Mobility Models for Mobile Ad hoc Networks," in Proc. of the ACM MobiCom'03, San Diego, USA, 2003, pp. 217-229.

[15] M. Kim, D. Kotz, and S. Kim, "Extracting a Mobility Model from Real User Traces," in Proc. of the IEEE INFOCOM'06, Barcelona, Spain, April 2006, pp. 1-13.

[16] V. Lenders, J. Wagner, and M. May, "Analyzing the Impact of Mobility in Ad Hoc Networks," in Proc. of the ACM REALMAN'06, Florence, Italy, 2006, pp. 39-46.

[17] C. Tuduce and T. Gross, "A Mobility Model Based on WLAN Traces and Its Validation," in Proc. of the IEEE INFOCOM'05, Miami, USA, 2005, pp. 664-674.

[18] A. Chaintreau, P. Hui, J. Crowcroft, C. Diot, R. Gass, and J. Scott, "Impact of Human Mobility on the Design of Opportunistic Forwarding Algorithms," in Proc. of the IEEE INFOCOM'06, Barcelona, Spain, April 2006, pp. 606-620.

[19] D. Lelescu, U. C. Kozat, R. Jain, and M. Balakrishnan, "Model T++:: an Empirical Joint Space-time Registration Model," in Proc. of the ACM MobiHoc'06, Florence, Italy, 2006, pp. 61-72.

[20] "CRAWDAD - Community Resource for Archiving Wireless Data At Dartmouth," URL http://crawdad.cs.dartmouth.edu/, acessado em 07/05/2007 